\title{
TABULATION OF PRIME PROJECTIONS OF LINKS IN THE THICKENED SURFACE OF GENUS 2 WITH NO MORE THAN 4 CROSSINGS ${ }^{1}$
}

\author{
A.A. Akimova \\ South Ural State University, Chelyabinsk, Russian Federation \\ E-mail: akimovaaa@susu.ru
}

In this article, we present the result of the first step of tabulation of prime links in the thickened surface of genus 2 that admit diagrams with no more than 4 crossings. Namely, we describe all three steps of tabulation of prime link projections in the surface of genus 2 with no more than 4 crossings. First, we define primality of a link projection in the surface of genus 2 . Second, we tabulate prime link projections in the surface of genus 2 with no more than 4 crossings. For this purpose, it is sufficient to consider graphs having special type and enumerate all possible embeddings of the graphs into the surface of genus 2 giving prime link projections. At this step, we prove some auxiliary statements to simplify enumeration of the embeddings. Finally, we show that all obtained projections are nonequivalent in the sense of homeomorphism of the surface of genus 2 onto itself. Our main result states that there exist exactly 15 pairwise nonequivalent prime link projections in the surface of genus 2 with no more than 4 crossings. Several new and known tricks allow rigorously theoretically prove the completeness of the obtained tabulation, as well as to keep the process within reasonable limits. Further, we intend to use the obtained table to classify prime diagrams, i.e. to obtain table of prime links.

Keywords: prime projection; link; thickened surface of genus 2; tabulation.

\section{Introduction}

In the knot theory, one of the oldest and the most important problems is to recognize a knot (or a link), i. e., to associate the considered object with a unique tabulated one. This problem involves the problem on complete classification of knots and links ordered taking into account some their properties. Many researchers worked in this aria during last 150 years. Most of the obtained classifications consider knots and links in the 3-dimensional sphere, see [1-3]. Recently, increasing interest in the theory of knots and links in arbitrary 3-manifolds (i. e., global knots and links) leads to tabulation of knots and links in manifolds different from the 3-dimensional sphere. However, in contrast to the case of knots and links in the 3-dimensional sphere, there is a gap between global knots and links in the sense of tabulation. In order to show this gap, compare presence of classifications of global knots and links.

As regards tabulation of global knots, note that knots in the solid torus [4] and the thickened Klein bottle [5], as well as prime knots in the lens spaces [6] are tabulated. In the knot theory, recent classifications consider only the so-called prime objects, which can not be obtained by some known operations from already tabulated objects. Knots in the thickened surfaces and virtual knots have been of particular interest during last 20 years. Hence, some classifications of such knots were also obtained. In particular, the works [7] and [8] present perfect classifications of virtual knots ordered taking into account the number of classical crossings and obtain a list of some characteristics of each knot. However, in these classifications, such important properties of a knot as primality and genus are not taken into account. Recall that genus of a virtual knot is the minimal genus of the thickened surface which can contain the considered knot. We propose to tabulate virtual knots taking into account both numerical characteristics, i. e. not only the number of classical crossings as usual, but also the genus of a knot, see the articles [9, 10] for classifications of prime knots in the thickened torus and the thickened surface of genus 2 , respectively. In a sense, such classifications can be considered as classifications of prime virtual knots of genus 1 and 2 , respectively. 


\section{Математика}

As regards tabulation of global links, note classifications of links in the projective space [11] and prime links in the thickened torus [12]. Also, note a classification of virtual links of special type, namely, alternating virtual links [13], see also [14] for the associated database, which include alternating virtual knots as well.

We begin tabulation of prime links in the thickened surface of genus 2. For this purpose, in this article, we present the result of the first step, i. e. we obtain a classification of prime projections of links in the surface of genus 2 with no more than 4 crossings. Our main result states that there exist exactly 15 pairwise nonequivalent (in the sense of homeomorphism of the surface of genus 2 onto itself) such projections. Further, we intend to use the obtained classification of prime projections in order to obtain classification of prime diagrams, i. e. classification of prime links.

The article is organized as follows. Section 1 describes required definitions and the main result of the article. Section 2 gives classification of prime projections of links in the surface of genus 2.

\section{Main Result}

A direct product of two copies of an 1-dimensional sphere $S^{1}$ is said to be a 2-dimensional torus $T=S^{1} \times S^{1}$. Hereinafter, for shortness, a 2-dimensional torus $T$ is called a torus $T$. As an example, consider a torus $T$ endowed with a pair "meridian-longitude" of $T$ shown in Fig. 1(a).

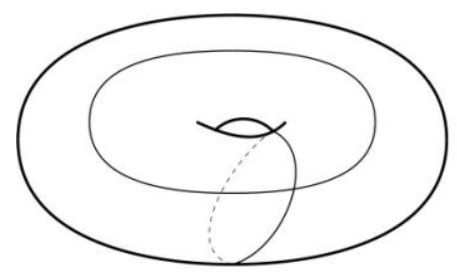

(a)

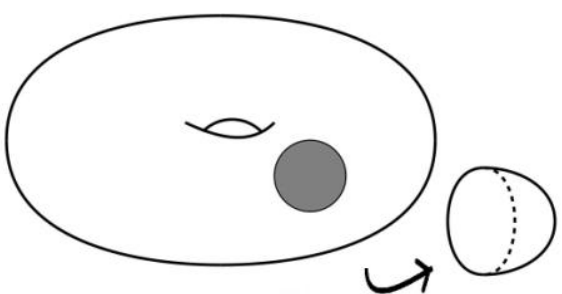

(b)

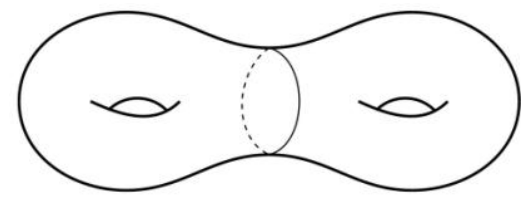

(c)

Fig. 1. (a) A torus $T$ endowed with a pair "meridian-longitude", (b) a torus $T^{o}$ with a hole and a disk $D^{2}$, (c) a 2-dimensional surface $T_{2}$ of genus 2 formed by gluing two copies of a torus $T^{o}$ with a hole

Let us remove the interior of a 2-dimensional disk $D^{2}$ from the initial surface $F$. As a result, we obtain a surface $F^{o}$ with a hole. Hereinafter, for shortness, a 2-dimensional disk $D^{2}$ is called a disk $D^{2}$. As an example, consider Fig. 1, $b$, where a torus $T^{o}$ with a hole is obtained from a torus $T$ by removing the interior of a disk $D^{2}$. Hereinafter, the notation of a surface with holes is endowed with the symbols of the form ${ }^{o}$, the number of which coincides with the number of holes.

A 2-dimensional surface $T_{2}$ of genus 2 is a surface obtained by identifying (gluing together) holes of two copies of a 2-dimensional torus $T^{o}$ with a hole, see Fig. 1, $c$. Here each surface $T^{o}$ is said to be a handle of a 2-dimensional surface $T_{2}$ of genus 2. Hereinafter, for shortness, a 2-dimensional surface $T_{2}$ of genus 2 is called a surface $T_{2}$.

We define types of simple closed curves, which can be considered in a surface $T_{2}$.

A simple closed curve $C \subset T_{2}$ is called cut, if the complement $T_{2} \backslash C$ consists of two components.

In the surface $T_{2}$, a cut curve $C$ can be either trivial, i. e. bounding a disk $D^{2}$, or not trivial. In the first case, the complement $T_{2} \backslash C$ is formed by a disk $D^{2}$ and a surface $T_{2}{ }^{o}$ with a hole. In the second case, the complement $T_{2} \backslash C$ is formed by two copies of a torus $T^{o}$ with a hole.

A simple closed curve $C \subset T_{2}$ is called not cut, if the complement $T_{2} \backslash C$ consists of the unique component. Namely, the complement $T_{2} \backslash C$ is a torus $T^{o o}$ with two holes. Two not cut simple closed curves

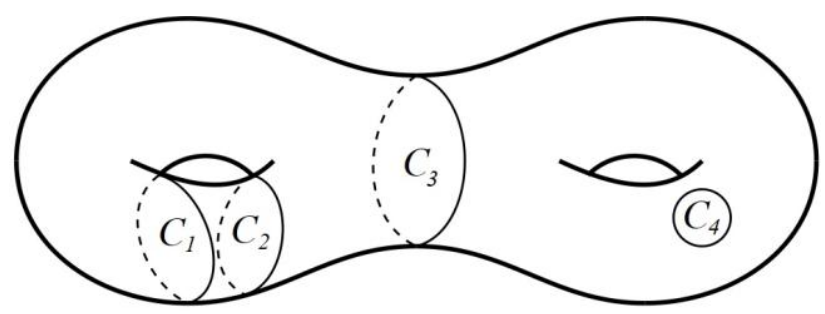

Fig. 2. Examples of curves in the surface $T_{2}$ $C_{1}, C_{2} \subset T_{2}$ are called parallel to each other, if the complement $T_{2} \backslash\left(C_{1} \mathrm{U} C_{2}\right)$ consists of two components, which are a torus $T^{o o}$ with two holes and an annulus $A$, i. e. a 2-dimensional sphere $S^{o o}$ with two holes.

Fig. 2 gives examples: $C_{1}, C_{2} \subset T_{2}$ are two not cut curves parallel to each other, while $C_{3}, C_{4} \subset T_{2}$ are not trivial and trivial cut curves, respectively. 
Consider a surface $T_{2}$ and an interval $I=[0,1]$. A 3-dimensional manifold homeomorphic to the direct product $T_{2} \times I$ is called a thickened surface of genus 2 .

A smooth embedding of a set of $m$ pairwise disjoint closed curves in the interior $\operatorname{Int}\left(T_{2} \times I\right)$ of the thickened surface $T_{2} \times I$ is said to be an m-component link in $T_{2} \times I$ and denoted by $L \subset T_{2} \times I$. In particular, a knot in $T_{2} \times I$ is obtained, if $m=1$, i. e. we consider a smooth embedding of a unique curve in $\operatorname{Int}\left(T_{2} \times I\right)$.

As in the classical case, a link $L$ in the thickened surface $T_{2} \times I$ can be represented by its diagram, which is defined by analogy with a classical link diagram except that $L$ is projected into the surface $T_{2}$ instead of a 2-dimensional sphere $S^{2}$.

A link projection in the surface $T_{2}$ is a diagram such that the crossings of the diagram contain no information about under/over-crossings. Hence, a link projection can be considered as an embedding of a regular graph of degree 4, i. e. valence of each vertex of the graph is equal to 4 . Vertices of $G$ are said to be crossings of $G$, connected components of the complement $T_{2} \backslash G$ are said to be faces of $G$, while each projection of a component of the link is said to be a component of $G$.

Two link projections $G$ and $G^{\prime}$ in the surface $T_{2}$ are called equivalent, if there exists a homeomorphism $f: T_{2} \rightarrow T_{2}$ such that $f(G)=G^{\prime}$.

An intersection point $P$ of two curves $C_{1}, C_{2} \subset T_{2}$ is said to be not transversal, if only two of four angles near $P$ are formed by small arcs of both curves $C_{1}$ and $C_{2}$, while the third and the forth angles are formed only by small arcs of the curve $C_{1}$ and $C_{2}$, respectively. Otherwise, i. e. if all four angles near $P$ are formed by both curves $C_{1}$ and $C_{2}$, the intersection point $P$ is said to be transversal. Note that there exist situations when $C_{1}$ and $C_{2}$ are the same curve $C$. In this case, we take into account the relative position of two small arcs of the curve $C$ as described above.

We define the following four types of link projections in the surface $T_{2}$.

1. The projection $G$ is said to be essential, if each face of $G$ is homeomorphic to a disk $D^{2}$.

2. The projection $G$ is said to be composite, if at least one of the following conditions holds.

(a) There exists a disk $D^{2} \subset T_{2}$ such that the boundary $\partial D^{2}$ intersects $G$ transversally exactly in two points, which are internal for two distinct edges of $G$, and at least one vertex of $G$ is inside $D^{2}$.

(b) There exist two parallel not cut simple closed curves $C_{1}, C_{2} \subset T_{2}$ and two distinct edges $e_{1}, e_{2}$ of $G$ such that for $i=1,2$ the curve $C_{i}$ intersects the edge $e_{i}$ transversally at the unique internal point, and both surfaces (a torus $T^{o o}$ with two holes and an annulus $A$ ) to which the curves divide the surface $T_{2}$ contain vertices of $G$.

(c) There exists not trivial cut simple closed curve $C$ and two distinct edges $e_{1}, e_{2}$ of $G$ such that for $i=1,2$ the curve $C$ intersects the edge $e_{i}$ transversally at the unique internal point, and both surfaces (two copies of a torus $T^{o}$ with a hole) to which the curve $C$ divides the surface $T_{2}$ contain vertices of $G$.

3. The projection $G$ is said to be not split, if each component of $G$ contains at least two crossings.

4. The projection $G$ is said to be prime, if $G$ is essential, not composite, not split and contains more than one component.

In this article, we consider only prime projections, i. e. only those that correspond to objects, which can not be obtained by some known operations from already tabulated ones.

Indeed, not essential projections correspond to links that can be found in the classifications of links in the 3-dimensional sphere $S^{3}$ [1-3], solid torus (thickened annulus $A \times I$ ) or thickened torus $T \times I$ [12]. Here we note that today there exist no classification of links in the solid torus, but, as well as in the case of knots, we consider the construction of such a classification as an independent problem, which is beyond the scope of our interests in this article.

In their turn, composite projections correspond to links, which can be obtained using already known links. Namely, composite projections of types (a)-(c) correspond to links, which can be obtained as sums of a classical link and a link in the thickened surface $T_{2} \times I$, a link in the thickened surface $T_{2} \times I$ and a link in the thickened torus $T \times I$, or two links in the thickened torus $T \times I$, respectively. Note that one of two terms in the sum can be a knot (see classifications obtained in $[1-4,9]$ ), since the result is a link anyway.

Finally, a split projection corresponds to a link, which can be considered as a trivial union of already tabulated links, while a link having the unique component is a knot.

Theorem 1. In the surface $T_{2}$, there exist exactly 15 pairwise nonequivalent prime link projections with no more than 4 crossings. The projections are shown in Fig. 5.

We prove Theorem 1 by three steps presented in Section 2 . 


\section{Математика}

\section{Proof of the main result}

In this section, we present main ideas of the tabulation of prime link projections. The tabulation is performed by three steps. First, Subsection 2.1 presents some auxiliary statements. Then, Subsection 2.2 enumerates all possible embeddings of the graphs into the surface $T_{2}$ giving prime projections. Finally, Subsection 2.3 shows that all obtained projections are pairwise nonequivalent.

\subsection{Some auxiliary statements}

Lemma 1. (Lemma 2 in [15]). Let $G \subset T_{2}$ be a prime projection with $n$ crossings, then $\mathrm{G}$ contains exactly $(n-2)$ faces.

Lemma 2. (Lemma 3 in [15]). There exist exactly 3 graphs with no more than 4 vertices whose embeddings into the surface $T_{2}$ can be prime projections, see graphs $a-c$ shown in Fig. 3 .

Lemma 3. Let $G \subset T_{2}$ be a prime link projection represented as a union of curves with $n$ intersection points. Then this union contains at most $(n-3)$ cut curves.

Proof. The statement is true in accordance with Lemma 1

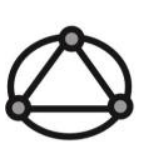

a

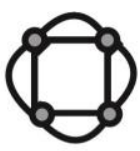

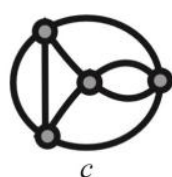

Fig. 3. The graphs of special type and the fact that each cut curve involves an additional face. This completes the proof of Lemma 3.

In this article, in order to obtain all the projections, we use the method of removing not transversal points [12] and the cutting technique [15].

Let $G \subset T_{2}$ be a prime link projection represented as a union $U$ of the curves $C_{i}, i=1,2, \ldots, m$ with $k$ not transversal intersection points.
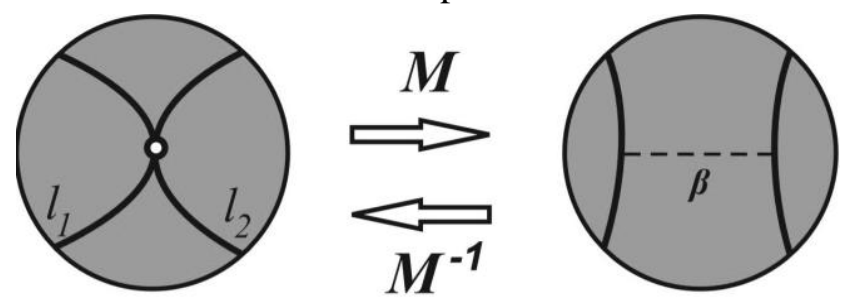

Fig. 4. Move $M$ removes a nontransversal point, while $M^{-1}$ is performed along the dotted line $\beta$ and creates the point

Let $l_{1}, l_{2}$ be small arcs containing a not transversal point of the projection $G$. We can remove the point by the move $M$ given in Fig. 4 . The dotted line $\beta$ shows how to perform the inverse move $M^{-1}$.

Remove each not transversal point of the projection $G$ by the move $M$. The obtained union $U^{k}$ of the same curves $C_{i}, i=1,2 \ldots, m$ includes only transversal points and is endowed with $k$ dotted lines $\beta$ to show where the move $M$ was performed. Of course, the initial projection $G$ can be obtained from $U^{k}$ by the inverse move $M^{-1}$ performed along each dotted line $\beta$, see Fig. 4.

Lemma 4. Let $G \subset T_{2}$ be a prime link projection obtained from the union $U^{k}$, which is endowed with $k$ dotted lines $\beta$. Then the union of $U^{k}$ and all $k$ dotted lines $\beta$ divide the surface $T_{2}$ into disks.

Proof. Otherwise, the projection $G$ is not essential, and we arrive at contradiction with the fact that the projection $G$ is prime. This completes the proof of Lemma 4.

\subsection{Construction of prime projections}

Lemma 5. All projections given in Fig. 5 can be obtained as embeddings of the graphs $a-c$ shown in Fig. 3. Namely, the graph $a$ involves the projection $3_{1}$, the graph $b$ involves the projections $4_{4}, 4_{11}$, and $4_{14}$, and the graph $c$ involves the projections $4_{1}-4_{3}, 4_{5}-4_{10}$ and $4_{12}, 4_{13}$.

Proof. In accordance with Lemma 2, all prime projections in the surface $T_{2}$ with no more than 4 crossings can be obtained as embeddings of the graphs $a-c$. In order to obtain all the projections, we represent an embedding of each graph as an union of a number of curves and enumerate all possible combinations of types of intersection points and curves.

Graph $a$. Suppose that the projection $G$ is an embedding of the graph $a$ in the surface $T_{2}$. The pairs of double edges form three curves in the surface $T_{2}$ such that each curve has the unique intersection point with each of two other curves. In accordance with Lemma 3, all curves are not cut.

Step 1. Assume that all three intersection points are not transversal, then, following [15], we arrive at contradiction with Lemma 1.

Step 2. Assume that exactly one of three intersection points is transversal, then, following [15], we obtain the knot projection only. 
Step 3. Assume that exactly two of three intersection points are transversal, then there exists a curve (e.g., $C_{1}$ ), which has the unique transversal intersection point with each of the rest curves. Hence, all three curves are not cut. We remove the unique not transversal point by the move $M$ and cut the surface $T_{2}$ along all the three curves.

If the not cut curves $C_{2}$ and $C_{3}$ are not parallel to each other, then we obtain an annulus $A$. In accordance with Lemma 4 , there is the unique way to construct a dotted line $\beta$ such that to connect two holes. Perform the inverse move $M^{-1}$ along the dotted line $\beta$ and obtain the projection $\mathbf{3}_{1}$.

If the not cut curves $C_{2}$ and $C_{3}$ are parallel to each other, then we obtain a disk $D^{2}$ and a torus $T^{o}$ with a hole, i. e. the projection contains more than one face, and we arrive at contradiction with Lemma 1.

Step 4. Assume that all three intersection points are transversal, then the projection contains a 3angle face, i. e. more than one face, and we arrive at contradiction with Lemma 1.

Graph $b$. Suppose that the projection $G$ is an embedding of the graph $b$ in the surface $T_{2}$. The pairs of double edges form four curves in the surface $T_{2}$ such that each curve has the unique intersection point with each of the two other curves and does not intersect the fourth curve.

Step 1. Assume that all four intersection points are not transversal, then, following [15], we arrive at contradiction with Lemma 1.

Step 2. Assume that exactly one of four intersection points is transversal, then, following [15], we obtain the knot projections only.

Step 3. Assume that exactly two of four intersection points are transversal. Note that these transversal points belong to different pairs of curves, since each pair of curves has no more than 1 common point. Hence, without loss of generality, we consider the curves $C_{1}$ and $C_{2}$ to be a pair "meridianlongitude" of one of the handles of the surface $T_{2}$, while the curves $C_{3}$ and $C_{4}$ form a pair "meridianlongitude" of another handle. We remove both not transversal points by the move $M$ and cut the surface $T_{2}$ along all the four curves to obtain an annulus $A$. There is the unique way to construct two dotted lines $\beta$ such that to connect two holes under the condition that there exists the unique endpoint of a dotted line $\beta$ on each curve $C_{i}, i=1,2,3,4$. Perform the inverse move $M^{-1}$ along each dotted line $\beta$ and obtain the projection $\mathbf{4}_{\mathbf{4}}$.

Step 4. Assume that exactly three of four intersection points are transversal, then there exists two curves (e.g., $C_{1}$ and $C_{2}$ ), each of which has the unique transversal intersection point with two other curves. Namely, $C_{1}$ has the unique transversal intersection point with $C_{2}$ and, e.g., $C_{3}$, while $C_{2}$ has the unique transversal intersection point with $C_{1}$ and $C_{4}$. Hence, all four curves $C_{i}, i=1,2,3,4$, are not cut and pairwise not parallel. We remove the unique not transversal point by the move $M$ and cut the surface $T_{2}$ along all the four curves to obtain a disk $D^{2}$. There is the unique way to construct a dotted line $\beta$ such that to connect the hole with itself under the condition that there exists the unique endpoint of a dotted line $\beta$ on both curves $C_{3}$ and $C_{4}$. Perform the inverse move $M^{-1}$ along the dotted line $\beta$ and obtain the projection $\mathbf{4}_{11}$.

Step 5. Assume that all four intersection points are transversal, then each curve has the unique transversal intersection point with two other curves. Hence, all four curves $C_{i}, i=1,2,3,4$, are not cut and pairwise not parallel. Obviously, we have the projection $\mathbf{4}_{\mathbf{1 4}}$.

Graph $c$. Suppose that the projection $G$ is an embedding of the graph $c$ in the surface $T_{2}$, then $G$ can be represented as a union of three curves such that the curves $C_{1}$ and $C_{2}$ have no common points, while the curve $C_{3}$ intersects each of them alternately. Hereinafter, without loss of generality, we consider the curve $C_{1}$ to be a representative of the curves $C_{1}$ and $C_{2}$. In accordance with Lemma 3 , there exists no more than one cut curve.

Step 1. Assume that all intersection points are not transversal.

Step 1.1. Assume that there exists no cut curves, then, following [15], we obtain the knot projections only.

Step 1.2. Assume that there exists a cut curve.

Step 1.2.1. Suppose that the cut curve is trivial. As mentioned above, in accordance with Lemma 3 , the rest curves are not cut. Also, note that the rest curves are not parallel to each other, otherwise as a result of cutting the surface $T_{2}$ along each curve $C_{i}, i=1,2,3$, we obtain a disk $D^{2}$, an annulus $A$ and a torus $T^{\circ o o}$ with three holes, i. e. the projection contains more than two faces, and we arrive at contradiction with Lemma 1. 


\section{Математика}

If the trivial cut curve is $C_{1}$, then, as mentioned above, the curves $C_{2}$ and $C_{3}$ are not cut and not parallel to each other. We remove each of four not transversal points by the move $M$ and cut the surface $T_{2}$ along all the three curves to obtain a disk $D^{2}$ and a sphere $S^{\text {ooooo }}$ with five holes. Note that the hole associated with the curve $C_{1}$ is connected by a dotted line $\beta$ with each of different holes associated with the curve $C_{3}$, since otherwise the projection contains more than two faces, and we arrive at contradiction with Lemma 1. Note that there is a misprint in [15], since we have the knot projection, if two holes associated with the curve $C_{2}$ are connected with different (but not the same as it was mentioned in [15]) holes associated with the curve $C_{3}$. Indeed, if each of two holes associated with the curve $C_{2}$ is connected by a dotted line $\beta$ with the same hole associated with the curve $C_{3}$, then we perform the inverse move $M^{-1}$ along each dotted line $\beta$ and obtain the projection $\mathbf{4}_{1}$.

If the trivial cut curve is $C_{3}$, then, as mentioned above, the curves $C_{1}$ and $C_{2}$ are not cut and not parallel to each other. We remove each of four not transversal points by the move $M$ and cut the surface $T_{2}$ along all the three curves to obtain a disk $D^{2}$ and a sphere $S^{\text {ooooo }}$ with five holes. There is the unique way to construct four dotted lines $\beta$ such that to connect each of holes associated with the curves $C_{1}$ and $C_{2}$ with the hole formed by the curve $C_{3}$ alternately. We perform the inverse move $M^{-1}$ along each dotted line $\beta$ and obtain the projection $\mathbf{4}_{5}$.

Step 1.2.2. Suppose that the cut curve is not trivial. Then this curve is $C_{3}$, since otherwise we have a not essential, i. e. nonprime, projection. For the same reason, the curves $C_{1}$ and $C_{2}$ are not parallel to each other. We remove each of four not transversal points by the move $M$ and cut the surface $T_{2}$ along all the three curves to obtain two copies of a sphere $S^{o o o}$ with three holes. In each $S^{o o o}$, there is the unique way to construct two dotted lines $\beta$ such that to connect the hole associated with the curve $C_{3}$ with each of the holes corresponded to the curves $C_{1}$ and $C_{2}$. Perform the inverse move $M^{-1}$ along each dotted line $\beta$ and obtain the projection $\mathbf{4}_{8}$.

Step 2. Assume that exactly one of four intersection points is transversal. Without loss of generality, we consider the curves $C_{1}$ and $C_{3}$ to be a pair "meridian-longitude" of one of the handles of the surface $T_{2}$, while the curve $C_{2}$ can be either cut or not cut. We remove all three not transversal points by the move $M$ and cut the surface $T_{2}$ along all the three curves.

Step 2.1. If the curve $C_{2}$ is cut, then $C_{2}$ is trivial, otherwise we have a not essential, i. e. nonprime, projection. Hence, as a result of cutting the surface $T_{2}$ along all the three curves, we obtain a torus $T^{o o}$ with two holes and a disk $D^{2}$. Note that there is the unique way to connect by a dotted line $\beta$ a fragment corresponded to the curve $C_{1}$ with a fragment corresponded to the curve $C_{3}$, since another way leads to a projection that contains more than two faces, and we arrive at contradiction with Lemma 1.

Step 2.1.1. If the hole associated with the curve $C_{2}$ is connected with different fragments of the curve $C_{3}$, then, following [15], we have the knot projection.

Step 2.1.2. If the hole associated with the curve $C_{2}$ is connected with the same fragment of the curve $C_{3}$, then we consider the following two possible situations.

First, there exists a fragment of the curve $C_{3}$ that contains no endpoints of the dotted lines $\beta$. In this case, we perform the inverse move $M^{-1}$ along each dotted line $\beta$ and obtain the projection $\mathbf{4}_{2}$.

Second, both fragments of the curve $C_{3}$ contain endpoints of the dotted lines $\beta$. In this case, we have a projection that contains more than two faces, and we arrive at contradiction with Lemma 1.

Step 2.2. If the curve $C_{2}$ is not cut, then as a result of cutting the surface $T_{2}$ along all the three curves, we obtain a sphere $S^{o o o}$ with three holes.

Step 2.2.1. If both holes associated with the curve $C_{2}$ are connected with the same fragment of the curve $C_{3}$, then, following [15], we have the knot projections both when there exists a fragment of the curve $C_{3}$ that contains no endpoints of the dotted lines $\beta$ and when such a fragment does not exists.

Step 2.2.2. If holes associated with the curve $C_{2}$ are connected with different fragments of the curve $C_{3}$, then, without loss of generality, we fix one of two possible fragments corresponded to the curve $C_{3}$ and consider all possible ways to connect by a dotted line $\beta$ this fragment with one of two fragments corresponded to the curve $C_{1}$. Since the curve $C_{3}$ should intersect the curves $C_{1}$ and $C_{2}$ alternately, then we have only two possible situations that are different in the sense of the choice of the fragment corresponded to the curve $C_{1}$. Then we perform the inverse move $M^{-1}$ along each dotted line $\beta$ and obtain the projections $\mathbf{4}_{\mathbf{3}}$ and $\mathbf{4}_{7}$.

Step 3. Assume that exactly two of four intersection points are transversal. 
First of all, we note the following obvious fact, which is very important for Steps 3-5 considered below. Namely, if both points that belong to the curve $C_{1}$ (or, due to symmetry, the curve $C_{2}$ ) are transversal, then the curve is not cut. Indeed, if the curve is either not trivial cut or trivial cut, then either the projection is not essential, i. e. nonprime, or the condition that the curve $C_{3}$ intersects the curves $C_{1}$ and $C_{2}$ alternately is not fulfilled, respectively.

Step 3.1. Suppose that both transversal points belong to the curve $C_{1}$. Then, as mentioned above, the curve $C_{1}$ is not cut.

Step 3.1.1. If the curve $C_{3}$ is cut, then the projection contains more than two faces, and we arrive at contradiction with Lemma 1.

Step 3.1.2. If the curve $C_{3}$ is not cut, then first of all note that the curve $C_{2}$ can not be not trivial cut, since otherwise either we have a not essential, i. e. nonprime projection, or the condition that the curve $C_{3}$ intersects the curves $C_{1}$ and $C_{2}$ alternately is not fulfilled. We remove both not transversal points by the move $M$ and cut the surface $T_{2}$ along all three curves. If the curve $C_{2}$ is not cut, then we obtain two copies of an annulus $A$. If the curve $C_{2}$ is trivial cut, then we obtain a sphere $S^{\circ o o}$ with three holes and a disk $D^{2}$. In both cases, in accordance with Lemma 4, there is the unique way to construct two dotted lines $\beta$. Perform the inverse move $M^{-1}$ along each dotted line $\beta$ and obtain the projections $\mathbf{4}_{9}$ and $\mathbf{4}_{\mathbf{1 0}}$, respectively.

Step 3.2. Suppose that each of the curves $C_{1}$ and $C_{2}$ contains the unique transversal point, then, following [15], we obtain the knot projections only.

Step 4. Assume that exactly three of four intersection points are transversal. Without loss of generality, we suppose that the curve $C_{1}$ contains two transversal points, while the curve $C_{2}$ contains one transversal point and one not transversal point. Hence, the curves $C_{2}$ and $C_{3}$ are not cut, since each of these curves contains the unique transversal point. As it was mentioned at Step 3, the curve $C_{1}$ is not cut. We remove the not transversal point by the move $M$ and cut the surface $T_{2}$ along all three curves to obtain a disk $D^{2}$. Due to symmetry, there is the unique way to construct a dotted line $\beta$ such that to connect the fragment corresponded to the curve $C_{2}$ with the fragment corresponded to the curve $C_{3}$. Perform the inverse move $M^{-1}$ along the dotted line $\beta$ and obtain the projection $\mathbf{4}_{6}$.

Step 5. Assume that all four intersection points are transversal. As it was mentioned at Step 3, both curves $C_{1}$ and $C_{2}$ are not cut.

Step 5.1. If the curve $C_{3}$ is trivial cut, then we arrive at contradiction with the condition that the curve $C_{3}$ intersects the curves $C_{1}$ and $C_{2}$ alternately.

Step 5.2. If the curve $C_{3}$ is not trivial cut, then we have the projection $\mathbf{4}_{12}$.

Step 5.3. If the curve $C_{3}$ is not cut, then we consider the following two possible situations.

Step 5.3.1. If the not cut curves $C_{1}$ and $C_{2}$ are parallel to each other, then the projection contains more than two faces, and we arrive at contradiction with Lemma 1.

Step 5.3.2. If the not cut curves $C_{1}$ and $C_{2}$ are not parallel to each other, then we obtain two projections: $\mathbf{4}_{13}$ and again $4_{12}$.

This completes the proof of Lemma 5 .

\subsection{Proof of the fact that all obtained projections are pairwise nonequivalent}

Lemma 6. All 15 projections shown in Fig. 5 are pairwise nonequivalent.

Proof. Let us associate each face of a projection with a natural number equal to the number of edges forming boundary of the face. Each face of a prime projection is homeomorphic to a disk. In accordance with Lemma 1, the number of faces of each projection shown in Fig. 5 is equal to 2 except for the projection $\mathbf{3}_{\mathbf{1}}$.

Associate each projection (with the exclusion of the projection $\mathbf{3}_{1}$ ) shown in Fig. 5 with an ordered set $\left\{(m) i_{1} i_{2} x\right\}$, where $m$ is the number of components, $i_{1}$ and $i_{2}$ are natural numbers, which are associated with the faces of the projection (taking in nondecreasing order), and $x$ is the graph such that the projection is an embedding of $x$ in the surface $T_{2}, x \in\{b, c\}$. Similarly, the projection $\mathbf{3}_{\mathbf{1}}$ is associated with the ordered set $\{(2) 12 a\}$.

Such ordered sets are sufficient to prove that all projections shown in Fig. 5 are pairwise nonequivalent except for the following 4 pairs: $\left(4_{1}, 4_{2}\right),\left(4_{5}, 4_{6}\right),\left(4_{8}, 4_{9}\right)$, and $\left(4_{12}, 4_{13}\right)$.

1. Projections $\left(4_{1}, 4_{2}\right)$ are nonequivalent, because only the first projection contains no self intersections of a component. The same is true for $\left(4_{5}, 4_{6}\right),\left(4_{8}, 4_{9}\right)$. 


\section{Математика}

2. Projections $\left(4_{12}, 4_{13}\right)$ are nonequivalent, because $4_{12}$ contains exactly 4 edges that are common for different 8 -gonal faces, while $4_{13}$ contains exactly 6 such edges.

Note that all tabulated projections are prime by construction.

This completes the proof of both Lemma 6 and Theorem 1.

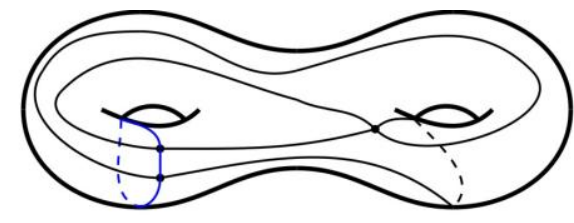

$3_{1}\{(2) 12 a\}$

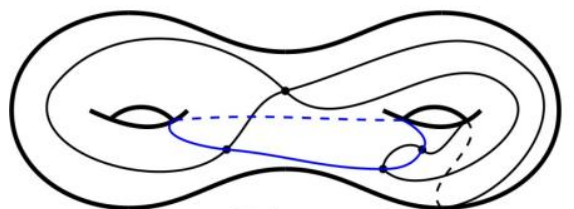

$4_{2}\{(2) 214 c\}$

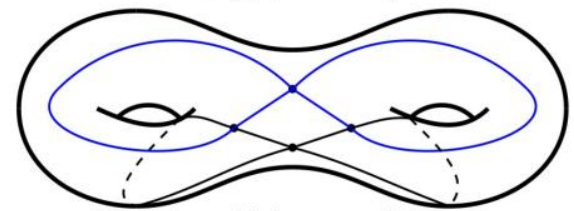

$4_{4}\{(2) 412 b\}$

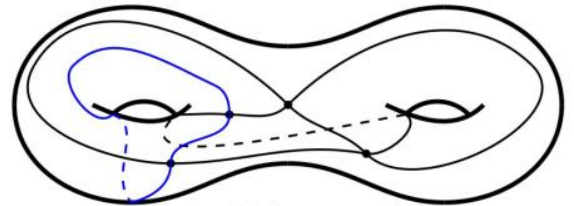

$4_{6}\{(2) 412 c\}$

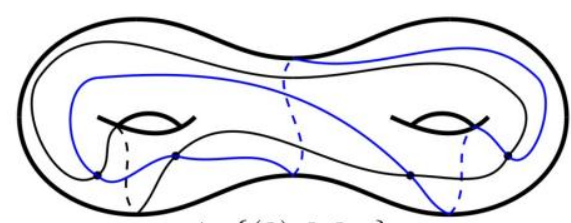

$4_{8}\{(2) 88 c\}$

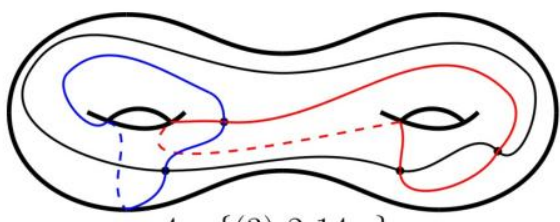

$4_{10}\{(3) 214 c\}$

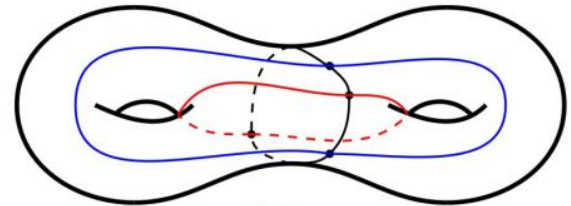

$4_{12}\{(3) 88 c\}$

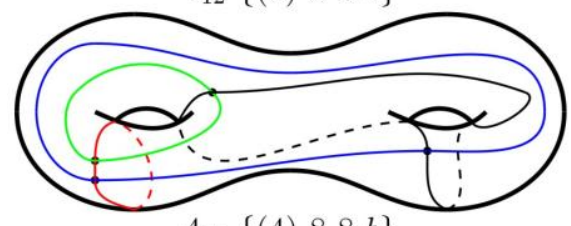

$4_{14}\{(4) 88$ b $\}$

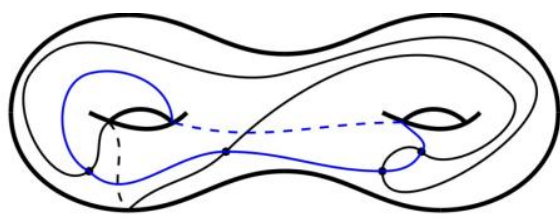

$4_{1}\{(2) 214 c\}$

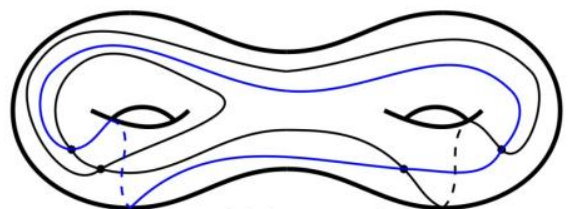

$4_{3}\{(2) 313 c\}$

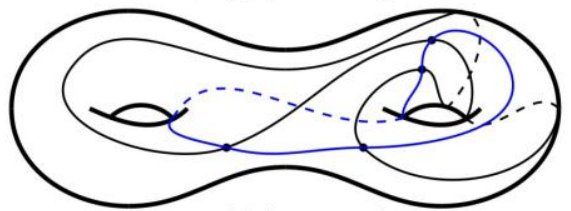

$4_{5}\{(2) 412 c\}$

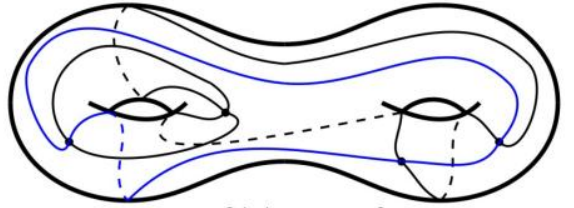

$4_{7}\{(2) 511 c\}$

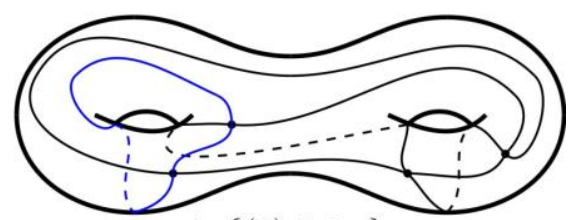

$4_{9}\{(2) 88 c\}$

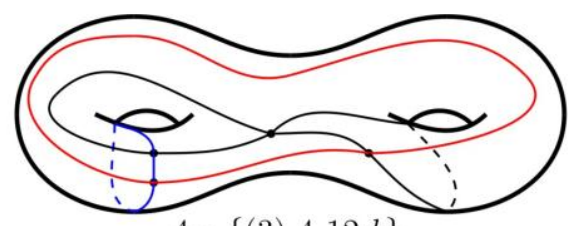

$4_{11}\{(3) 412$ b $\}$

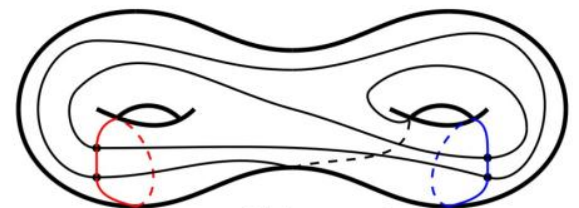

$4_{13}\{(3) 88 c\}$

Fig. 5. Prime link projections in the surface $T_{2}$ with no more than 4 crossings 


\section{References}

1. Hoste J., Thistlethwaite M., Weeks J. The first 1,701,936 knots. The Mathematical Intelligencer, 1998, Vol. 20, Iss. 4, pp. 33-48. DOI: 10.1007/BF03025227

2. Rolfsen D. Knots and Links. Berkeley, CA: Publish or Perish, 1976.

3. Bar-Natan D. The Knot Atlas, http://katlas.org/wiki/Main_Page.

4. Gabrovšek B., Mroczkowski M. Knots in the Solid Torus up to 6 Crossings. Journal of Knot Theory and Its Ramifications, 2012, Vol. 21, no. 11, pp. 1250106-1-1250106-43. DOI: $10.1142 / \mathrm{S} 0218216512501064$

5. Matveev S.V., Nabeeva L.R. Tabulating knots in the thickened Klein bottle. Siberian Mathematical Journal, 2016, Vol. 57, Iss. 3, pp. 542-548. DOI: 10.1134/S0037446616030174

6. Gabrovšek B. Tabulation of Prime Knots in Lens Spaces. Mediterranean Journal of Mathematics, 2017, Vol. 14, no. 88. DOI: 10.1007/s00009-016-0814-5

7. Green J. A table of virtual knots, https://www.math.toronto.edu/drorbn/Students/GreenJ/

8. Stenlund E. Classification of virtual knots, http://evertstenlund.se/knots/Virtual\%20Knots

9. Akimova A.A., Matveev S.V. Virtual Knots Having at most Five Classical Crossings. Journal of Knot Theory and Its Ramifications, 2014, Vol. 23, no. 6, pp. 1450031-1-1450031-19. DOI: $10.1142 / \mathrm{S} 021821651450031 \mathrm{X}$

10. Akimova A.A. Classification of prime knots in the thickened surface of genus 2 having diagrams with at most 4 crossings. Journal of Computational and Engineering Mathematics, 2020, Vol. 7, no. 1. pp. 32-46. DOI: $10.14529 /$ jcem 200103

11. Drobotukhina Yu.V. Classification of links in $\mathrm{RP}^{3}$ with at most 6 crossings, Geometry and topology. Part 1. Zap. Nauchn. Sem. LOMI, Vol. 193, Leningrad, Nauka publ., 1991, pp. 39-63. [Drobotukhina Yu.V. Classification of links in $\mathrm{RP}^{3}$ with at most six crossings, Advances in Soviet Mathematics, 1994, Vol. 18, no. 1, pp. 87-121].

12. Akimova A.A., Matveev S.V., Tarkaev V.V. Classification of Links of Small Complexity in the Thickened Torus. Proceedings of the Steklov Institute of Mathematics, 2018, Vol. 303, Suppl. 1, pp. 1224. DOI: $10.1134 / \mathrm{s} 008154381809002 \mathrm{x}$

13. Zinn-Justin P., Zuber J. B. Matrix Integrals and the Generation and Counting of Virtual Tangles and Links. Journal of Knot Theory and Its Ramifications, 2004, Vol. 13, no. 3, pp. 325-355. DOI: $10.1142 / \mathrm{S} 0218216504003172$

14. Zinn-Justin P. Alternating Virtual Link Database. https://www.lpthe.jussieu.fr/pzinn/virtlinks/

15. Akimova A.A. Classification of prime projections of knots in the thickened torus of genus 2 with at most 4 crossings. Bulletin of the South Ural State University Series "Mathematics. Mechanics. Physics", 2020, Vol. 12, no.1, pp. 5-13. DOI: 10.14529/mmph200101

Received July 7, 2020

\section{ТАБУЛЯЦИЯ ПРИМАРНЫХ ПРОЕКЦИЙ ЗАЦЕПЛЕНИЙ В УТОЛЩЕННОМ КРЕНДЕЛЕ РОДА 2 С НЕ БОЛЕЕ ЧЕМ 4 ПЕРЕКРЕСТКАМИ}

\section{А.А. Акимова}

Южно-Уральский государственный университет, г. Челябинск, Российская Федерация E-mail: akimovaaa@susu.ru

Мы представляем первый этап табуляции примарных зацеплений в утолщенном кренделе рода 2, имеющих диаграммы с не более чем 4 перекрестками, а именно, приводятся все три этапа классификации примарных проекций зацеплений на кренделе рода 2, имеющих не более чем 4 перекрестка. Сначала мы вводим понятие примарности проекции зацепления на кренделе рода 2. Затем мы строим таблицу примарных проекций зацеплений на кренделе рода 2, имеющих не бо- 


\section{Математика}

лее чем 4 перекрестка. Для этого мы перечисляем графы специального вида и рассматриваем все возможные вложения этих графов в крендель рода 2, которые приводят к примарым проекциям. С целью сокращения перечисления таких вложений мы доказываем несколько вспомогательных утверждений. В конце работы мы показываем различность всех полученных проекций в смысле гомеоморфизма кренделя рода 2 на себя. Наш основной результат состоит в том, что существует ровно 15 попарно неэквивалентных примарных проекций зацеплений на кренделе рода 2 с не более чем 4 перекрестками. Ряд новых и известных приемов позволили строго теоретически доказать полноту построенной таблицы и удержать процесс перебора в разумных пределах. Полученная классификация проекций будет использована для проведения классификации примарных диаграмм, т. е. построения таблицы примарных зацеплений.

Ключевые слова: примарная проекция; защепление; утолщенный крендель рода 2; таблица.

\section{Литература}

1. Hoste, J. The first 1,701,936 knots / J. Hoste, M. Thistlethwaite, J. Weeks // The Mathematical Intelligencer. - 1998. - Vol. 20, Iss. 4. - pp. 33-48

2. Rolfsen, D. Knots and Links / D. Rolfsen. - Berkeley, CA: Publish or Perish, 1976.

3. Bar-Natan, D. The Knot Atlas, http://katlas.org/wiki/Main_Page.

4. Gabrovšek, B. Knots in the Solid Torus up to 6 Crossings / B. Gabrovšek, M. Mroczkowski // Journal of Knot Theory and Its Ramifications. - 2012. - Vol. 21, no. 11. - P. 1250106-1-1250106-43.

5. Матвеев, С.В. Табулирование узлов в утолщенной бутылке Клейна / С.В. Матвеев, Л.Р. Набеева // Сиб. матем. журн. - 2016. - Т. 57, № 3. - С. 688-696.

6. Gabrovšek, B. Tabulation of Prime Knots in Lens Spaces / B. Gabrovšek // Mediterranean Journal of Mathematics. - 2017. - Vol. 14, no. 88.

7. Green, J. A table of virtual knots. - https://www.math.toronto.edu/drorbn/Students/GreenJ/

8. Stenlund, E. Classification of virtual knots. - http://evertstenlund.se/knots/Virtual\%20Knots.pdf

9. Akimova, A.A. Classification of Genus 1 Virtual Knots Having at most Five Classical Crossings / A.A. Akimova, S.V. Matveev // Journal of Knot Theory and Its Ramifications. - 2014. - Vol. 23, no. 6. P. 1450031-1-1450031-19.

10. Akimova, A.A. Classification of prime knots in the thickened surface of genus 2 having diagrams with at most 4 crossings / A.A. Akimova // Journal of Computational and Engineering Mathematics. - 2020. - Vol. 7, no. 1. - P. 32-46.

11. Дроботухина, Ю.В. Классификация зацеплений в $\mathrm{RP}^{3}$ с небольшим числом точек скрещивания / Ю.В. Дроботухина // Зап. научн. сем. ЛОМИ. - 1991. - Т. 193. - С. 39-63.

12. Акимова, А.А. Классификация зацеплений малой сложности в утолщенном торе / А.А. Акимова, С.В. Матвеев, В.В.Таркаев // Труды института математики и механики УрО РАН. - 2017. - Т. 23, Вып. 4. - С. 18-31.

13. Zinn-Justin, P. Matrix Integrals and the Generation and Counting of Virtual Tangles and Links / P. Zinn-Justin, J.B. Zuber // Journal of Knot Theory and Its Ramifications. - 2004. - Vol. 13, no. 3. P. 325-355.

14. Zinn-Justin, P. Alternating Virtual Link Database / P. Zinn-Justin // https://www.lpthe.jussieu.fr/pzinn/virtlinks/

15. Akimova, A.A. Classification of Prime Projections of Knots in the Thickened Torus of Genus 2 with at most 4 Crossings / A.A. Akimova // Вестник ЮУрГУ. Серия «Математика. Механика. Физика». - 2020. - Т. 12, № 1. - С. 5-13.

Поступила в редакцию 7 июля 2020 2. 